\title{
Periodic coefficients and random Fibonacci sequences
}

\author{
Karyn McLellan \\ Department of Mathematics and Statistics \\ Dalhousie Unviersity \\ Halifax, Nova Scotia, Canada \\ mkaryn@mathstat.dal.ca
}

Submitted: Mar 15, 2013; Accepted: Nov 27, 2013; Published: Dec 17, 2013

Mathematics Subject Classifications: 11B39, 05A05, 15B36

\begin{abstract}
The random Fibonacci sequence is defined by $t_{1}=t_{2}=1$ and $t_{n}= \pm t_{n-1}+$ $t_{n-2}$, for $n \geqslant 3$, where each \pm sign is chosen at random with probability $P(+)=$ $P(-)=\frac{1}{2}$. Viswanath has shown that almost all random Fibonacci sequences grow exponentially at the rate $1.13198824 \ldots$ We will consider what happens to random Fibonacci sequences when we remove the randomness; specifically, we will choose coefficients which belong to the set $\{1,-1\}$ and form periodic cycles. By rewriting our recurrences using matrix products, we will analyze sequence growth and develop criteria based on eigenvalue, trace and order for determining whether a given sequence is bounded, grows linearly or grows exponentially. Further, we will introduce an equivalence relation on the coefficient cycles such that each equivalence class has a common growth rate, and consider the number of such classes for a given cycle length.
\end{abstract}

Keywords: random Fibonacci sequence, growth rate, periodic, matrix trace

\section{Introduction}

In 1999, Viswanath [19] introduced a random component to the Fibonacci sequence, such that instead of adding consecutive terms, we either add or subtract with equal probability. He was able to prove some remarkable results about this new sequence.

Definition 1. The random Fibonacci sequence $\left\{t_{n}\right\}$ has initial terms $t_{1}=t_{2}=1$ and is given by the recurrence

$$
t_{n}= \pm t_{n-1} \pm t_{n-2}
$$

for $n \geqslant 3$, where each \pm sign is chosen independently with probability $P(+)=P(-)=\frac{1}{2}$. 
Viswanath instead used the sequence

$$
t_{n}= \pm t_{n-1}+t_{n-2}
$$

which because of symmetry in the probabilities, is equivalent to that defined in (1). (We could equally well have placed the \pm sign before the latter term.)

Theorem 2 (Viswanath). For almost all random Fibonacci sequences, the growth rate is given by

$$
\lim _{n \rightarrow \infty}\left|t_{n}\right|^{\frac{1}{n}}=1.13198824 \ldots
$$

Viswanath's result is counterintuitive because we might expect that with an equal number of additions and subtractions (on average), the terms would eventually balance out to zero. Or, it could be possible that they jump around so chaotically that no limit in the growth rate is reached.

Viswanath was only able to calculate eight decimal places of this constant (see [20, A078416]) through extensive computations of upper and lower bounds. In 2007, Bai [1] extended the constant by five decimal places to $1.1319882487943 \ldots$. . There is no known closed form or analytic expression for Viswanath's constant and nothing else is known about its nature, although it is reasonable to conjecture that it is irrational and also transcendental.

Viswanath's problem was considered in greater generality by Furstenberg [7] in 1963, when he studied the asymptotic behaviour of the sequence defined by the recurrence $x_{n}=\alpha_{n} x_{n-1}+\beta_{n} x_{n-2}$, where $\left(\alpha_{n}, \beta_{n}\right)$ form a sequence of i.i.d. random vectors. From Furstenberg's work, Viswanath was able to conclude that almost all random Fibonacci sequences $\left\{t_{n}\right\}$ grow exponentially. Many after Viswanath have varied and generalized his ideas. Embree and Trefethen [6] introduced a parameter to the recurrence and found the critical value at which growth turns to decay, and Janvresse, Rittaud and de la Rue (see [10] and [11]) also introduced a parameter, as well as let $0<p \leqslant 1$.

Random matrix products and random Fibonacci-type sequences arise in a wide range of fields, in particular, those involving one-dimensional disordered systems. For example, applications include randomly coupled harmonic oscillators, quantum mechanics of an electron in one dimension, population evolution (Bai [2]), resistance in an electrical network (Colman and Rodgers [4]), and materials with irregular atomic structure (Devlin [5]).

The aim of this paper is to remove the randomness from Viswanath's work. Instead of tackling the growth of a random Fibonacci sequence using random matrix theory or other stochastic methods, we use matrix products obtained from periodic sequences. McGuire [13] independently had the idea to study deterministic matrix products and to observe the behaviour of the corresponding sequences. He gave necessary conditions for such a sequence to be periodic, as well as the possible periods of the sequences.

In Section 2 we introduce the framework for the main results of this paper, including product matrices, and periodic coefficient sequences and their growth rates, along with several examples. To do this we must first consider the growth of a general second order 
linear recurrence. In Section 3 we study the trace and determinant of a product matrix, and determine how these criteria can be used to classify periodic coefficient sequences according to growth type. We also look at the connection between growth type and order of a product matrix, an idea which was considered by McGuire [13]. In Section 4 we examine some methods used to approximate Viswanath's constant, and further, consider an equivalence relation on coefficient cycles which allows us to reduce the computations required for such an approximation.

\section{Growth Types of Periodic Coefficient Sequences}

Our aim in this section is to remove the randomness from Viswanath's random Fibonacci sequence by forming sequences according to a fixed pattern. These sequences will have growth rates that differ from the almost sure value $1.13198824 \ldots$. We begin this task by introducing some definitions, which allow us to formulate the "non-random Fibonacci sequence".

Definition 3. (a) A coefficient cycle of length $n$ is an $n$-tuple $\sigma_{n}=\left(s_{1}, \ldots, s_{n}\right)$, where $s_{j} \in\{+,-\}$ for $1 \leqslant j \leqslant n$.

(b) The periodic coefficient sequence for $\sigma_{n}$ is given by the recursion in Equation (2) for $i \geqslant 3$, where $t_{1}=t_{2}=1$ and each \pm sign is chosen according to $\sigma_{n}$ as follows:

$$
t_{i}=s_{1+(i-3) \bmod n} t_{i-1}+t_{i-2} .
$$

The index $j$ of $s_{j}$ takes on the values $1, \ldots, n$, and then cycles back through as $i$ increases. The following motivating example demonstrates the different types of behaviours of a periodic coefficient sequence. (For simplicity of notation, we will remove the commas.)

Example 4. Let $\sigma_{3}=(++-)$. Generating the periodic coefficient sequences gives

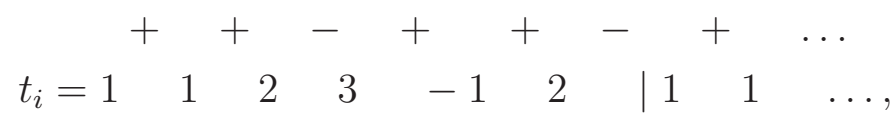

where the bar denotes repetition. The period of repetition is 6 , which is a multiple of $n=3$. If we let $\sigma_{4}=(++--)$, the corresponding sequence is

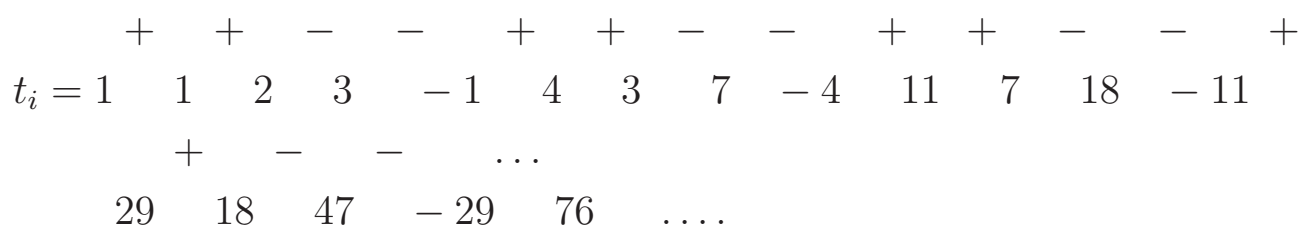

and it appears as if the sequence is unbounded in absolute value. Also, the coefficient cycle $\sigma_{6}=(+++---)$ generates the periodic coefficient sequence

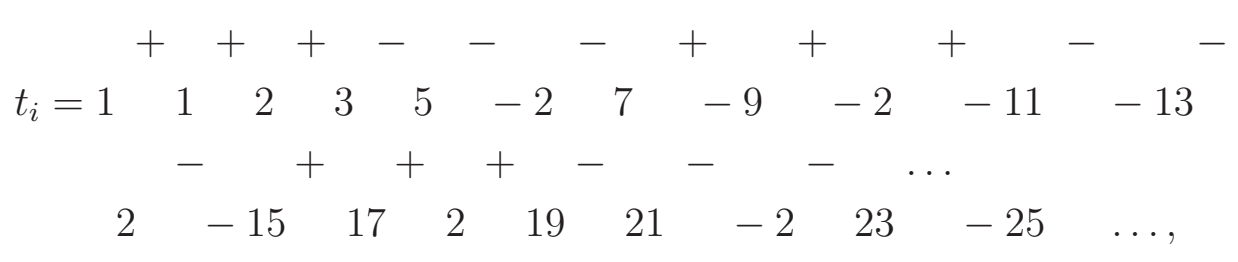


which appears to be growing linearly in absolute value, with every third term equal to 2.

Upon inspection, there is no obvious connection between the coefficient cycles $\sigma_{n}$ and the growth type. In order to understand the behaviour of our periodic coefficient sequences, we can represent the recurrence given in Equation (3) as a matrix equation for $i \geqslant 3$ as follows:

$$
\left(\begin{array}{c}
t_{i-1} \\
t_{i}
\end{array}\right)=\left(\begin{array}{cc}
0 & 1 \\
1 & \pm 1
\end{array}\right)\left(\begin{array}{c}
t_{i-2} \\
t_{i-1}
\end{array}\right)
$$

where the vector $\left(t_{i-2}, t_{i-1}\right)^{T}$ is multiplied by a member of

$$
\left\{A=\left(\begin{array}{ll}
0 & 1 \\
1 & 1
\end{array}\right), B=\left(\begin{array}{cc}
0 & 1 \\
1 & -1
\end{array}\right)\right\},
$$

according to the value of the term $s_{1+(i-3) \bmod n}$ in the coefficient cycle $\sigma_{n}$ : $A$ for + and $B$ for - . Note that for Viswanath's random Fibonacci sequence given by Equation (2), the matrix equation is the same, but we choose one of $A$ or $B$ with probability $\frac{1}{2}$. Janvresse et al. [10] study the random Fibonacci sequence $t_{i}=t_{i-1} \pm t_{i-2}$, where the \pm sign is shifted, as mentioned in Section 1. This results in a matrix recurrence using $A, B=\left(\begin{array}{cc}0 & 1 \\ \pm 1 & 1\end{array}\right)$. Also, for the regular Fibonacci sequence, we choose $A$ at every step. Iterating Equation (4) for $i=n+2$, and incorporating initial values, gives

$$
\left(\begin{array}{c}
t_{n+1} \\
t_{n+2}
\end{array}\right)=M_{n} M_{n-1} \cdots M_{1}\left(\begin{array}{l}
1 \\
1
\end{array}\right),
$$

where $M_{j} \in\{A, B\}$ for $1 \leqslant j \leqslant n$, and is chosen by $s_{j}$ in $\sigma_{n}=\left(s_{1}, \ldots, s_{n}\right)$.

Definition 5. Given $M_{j}$ for $1 \leqslant j \leqslant n$ as in (5), we define the product matrix $P_{n}$ associated with the coefficient cycle $\sigma_{n}$ to be $P_{n}:=M_{1} \cdots M_{n}$.

We will later see that reversing the order of the matrices in Equation (5) to define $P_{n}$ is permissible when analyzing the growth of the associated sequence, so we write our product matrix with increasing indices so that it better reflects the pattern in the corresponding coefficient cycle. We will still need to use the form in Equation (5) when finding explicit sequence terms, however.

Since $\operatorname{det}(A)=\operatorname{det}(B)=-1$, all matrices $P_{n}$ are unimodular. For $n$ even or odd we have $\operatorname{det}\left(P_{n}\right)=1$ or -1 , respectively. It is straightforward to show that the matrices $A$ and $B$ form a multiplicative group

$$
G:=\langle A, B\rangle \leqslant \mathrm{S}^{*} \mathrm{~L}(2, \mathbb{Z})=\mathrm{GL}(2, \mathbb{Z}),
$$

where $\mathrm{S}^{*} \mathrm{~L}(2, \mathbb{Z})$ is the extension of the special linear group to unimodular matrices. This is not a free group because various relations, such as $A A B A A B=I$ exist between the matrices. We have the following characterization of elements $P_{n} \in K$, where $K:=$ $G \cap \mathrm{SL}(2, \mathbb{Z})$.

Proposition 6. The group $K<G$ is composed of exactly those elements $P_{n}=\left(\begin{array}{ll}a & b \\ c & d\end{array}\right) \in$ $\mathrm{SL}(2, \mathbb{Z})$ with $P_{n} \equiv\left(\begin{array}{ll}1 & 0 \\ 0 & 1\end{array}\right),\left(\begin{array}{ll}0 & 1 \\ 1 & 1\end{array}\right)$, or $\left(\begin{array}{ll}1 & 1 \\ 1 & 0\end{array}\right)$ when we take $a, b, c, d$ modulo 2 . 
We will now take a closer look at the three types of growth demonstrated by the periodic coefficient sequences. To do this, we will first need to consider the growth of a general second order linear recurrence relation

$$
a_{n}=u a_{n-1}+v a_{n-2},
$$

for $n \geqslant 3$, where $a_{1}, a_{2}, u, v \in \mathbb{Z}$.

Definition 7. We say a sequence $\left\{a_{n}\right\}$ defined by a second order linear recurrence has exponential growth if

$$
\lim _{n \rightarrow \infty}\left|a_{n}\right|^{\frac{1}{n}}=c
$$

with $c>1$. We call $c$ the growth rate of the sequence. We can similarly define the growth rate as

$$
\lim _{n \rightarrow \infty}\left|\frac{a_{n+1}}{a_{n}}\right|=c
$$

when this limit exists. We say a sequence $\left\{a_{n}\right\}$ defined by a second order linear recurrence has linear growth if the terms can be written as

$$
a_{n}=p n+q,
$$

for some $p, q \in \mathbb{Z}, p \neq 0$. We say a sequence $\left\{a_{n}\right\}$ defined by a second order linear recurrence has bounded growth if for all $n$ we have that $\left|a_{n}\right| \leqslant q$ for some $q \in \mathbb{Z}$.

Bajaj [3], for example, has shown that for a positive sequence $\left\{a_{n}\right\}$, if the limits in Equations (7) and (8) both exist, then they are equal.

The growth type of a general second order linear recurrence can be determined by the eigenvalues of the companion matrix, which is used to represent the recurrence as

$$
\left(\begin{array}{c}
a_{n-1} \\
a_{n}
\end{array}\right)=\left(\begin{array}{ll}
0 & 1 \\
v & u
\end{array}\right)\left(\begin{array}{l}
a_{n-2} \\
a_{n-1}
\end{array}\right)
$$

From the characteristic equation $x^{2}-u x-v=0$ we find the eigenvalues $\lambda_{1}$ and $\lambda_{2}$ in terms of $u$ and $v$, where we assume $\left|\lambda_{1}\right| \geqslant\left|\lambda_{2}\right|$, i.e., $\lambda_{1}$ is the dominant eigenvalue. Note that the characteristic equation can also be obtained from the recurrence given in Equation (6).

Using the well known facts (for example, see Vajda [17, p. 18]) that the closed-form expression for the term $a_{n}$, with $a_{n} \geqslant 1$ is given as

$$
a_{n}=\alpha \lambda_{1}^{n}+\beta \lambda_{2}^{n}
$$

for $\lambda_{1} \neq \lambda_{2}$, where $\alpha$ and $\beta$ rely on the initial values $a_{1}$ and $a_{2}$, and

$$
a_{n}=(\alpha+\beta n) \lambda_{1}^{n}
$$

for $\lambda_{1}=\lambda_{2}$, we can deduce that the growth of a general second order linear recurrence must have one of the growth types bounded, linear or exponential. Further, we have the following proposition. 
Proposition 8. The growth of a second order linear recurrence sequence, where $\lambda_{1}$ and $\lambda_{2}$ are eigenvalues of the companion matrix, is

i. exponential if and only if $\left|\lambda_{1}\right|>1$,

ii. linear if and only if $\lambda_{1}$ and $\lambda_{2}$ are equal roots of unity,

iii. bounded if and only if $\left|\lambda_{1}\right|,\left|\lambda_{2}\right| \leqslant 1$ where the eigenvalues are not equal roots of unity, with the exception of the following cases. The growth is also bounded if $a_{1}=a_{2}$ with $\lambda_{1}$ or $\lambda_{2}=1 ; a_{1}=-a_{2}$ with $\lambda_{1}$ or $\lambda_{2}=-1 ; a_{2}=0$ with $\lambda_{1}$ or $\lambda_{2}=0 ;$ or $a_{1}=a_{2}=0$. The growth rate of an exponentially growing sequence given by $\left|\lambda_{1}\right|$.

Proofs of the preceding results and further details can be found in McLellan [16].

We now want to apply this information to our periodic coefficient sequences. The key is to think of a periodic coefficient sequence for $\sigma_{n}$ as being composed of $n$ subsequences. An example here will be helpful.

Example 9. The periodic coefficient sequence for $\sigma_{4}=(++--)$ is listed vertically to create four subsequences which appear to be growing exponentially.

$\begin{array}{rrrrrr}1, & -1, & -4, & -11, & -29, & \ldots \\ 1, & 4, & 11, & 29, & 76, & \ldots \\ 2, & 3, & 7, & 18, & 47, & \ldots \\ 3, & 7, & 18, & 47, & 123, & \ldots\end{array}$

We can see that each is growing according to the recurrence relation

$$
t_{n}=3 t_{n-1}-t_{n-2} \text {. }
$$

The product matrix in this example is $P_{4}=A A B B=\left(\begin{array}{cc}0 & 1 \\ -1 & 3\end{array}\right)$. The characteristic equation is $x^{2}-3 x+1=0$, which is also what we get from Equation (9). The eigenvalues are $\frac{3 \pm \sqrt{5}}{2}$, which indicate exponential growth by Proposition 8 .

In this example, $P_{4}$ is in the form of a companion matrix, which, however, is not always the case. The characteristic equation obtained from a product matrix $P_{n}=\left(\begin{array}{ll}a & b \\ c & d\end{array}\right)$ is $x^{2}-(a+d) x+(a d-b c)=0$. Comparing with the form of the characteristic equation $x^{2}-u x-v=0$, we see that

$$
\begin{gathered}
u=a+d=\operatorname{tr}\left(P_{n}\right), \\
v=-(a d-b c)=-\operatorname{det}\left(P_{n}\right),
\end{gathered}
$$

where $u, v \in \mathbb{Z}$ because we have seen the matrices $P_{n}$ belong to $\mathrm{S}^{*} \mathrm{~L}(2, \mathbb{Z})$. Note also that this tells us that $v= \pm 1$, which we have seen. Using this together with the fact that the product of eigenvalues is the determinant of a matrix, we can rewrite Proposition 8 as follows. 
Theorem 10. Given a product matrix $P_{n}$ with eigenvalues $\lambda_{1}$ and $\lambda_{2}$, the growth of a given subsequence is

i. exponential if and only if $\left|\lambda_{1}\right|>1$,

ii. linear if and only if $P_{n} \neq \pm I$ and the eigenvalues $\lambda_{1}, \lambda_{2}$ are both 1 or both -1 ,

iii. bounded if and only if $P_{n}= \pm I$ or $\lambda_{1}, \lambda_{2}$ are distinct roots of unity,

with the following exceptions. The growth is also bounded if $\lambda_{1}=1$ with $a_{1}=a_{2} ; \lambda_{1}=-1$ with $a_{1}=-a_{2}$; or $a_{1}=a_{2}=0$. The growth rate of an exponentially growing subsequence is $\left|\lambda_{1}\right|$.

In order to generate the first two subsequences in Example 9, we can use the matrix $B B A A$ (the reverse product of $P_{4}$ ) multiplied by the initial value vector, as given in Equation (5) and mentioned in the remark after Definition 5. In order to generate the other subsequences, we must rotate the terms in our product matrix and use the corresponding initial value vector.

Proposition 11. Given a coefficient cycle $\sigma_{n}=\left(s_{1}, \ldots, s_{n}\right)$, with $n \geqslant 1$, rotating the entries (to the right) by $k$, where $0 \leqslant k \leqslant n-1$, gives a new cycle

$\sigma_{n}^{\prime}=\left(s_{n-k+1}, \ldots, s_{n}, s_{1}, \ldots, s_{n-k}\right)$ with corresponding product matrix having the same characteristic equation as the original product matrix.

It is a straightforward calculation to show that the trace and determinant, and hence characteristic equation are unchanged by such a rotation.

Corollary 12. Given a coefficient cycle $\sigma_{n}=\left(s_{1}, \ldots, s_{n}\right)$, with $n \geqslant 1$, the corresponding periodic coefficient sequence can be broken down into $n$ subsequences, each of which grows according to the same second order linear recurrence relation.

Example 13. The periodic coefficient sequence for $\sigma_{6}=(+++---)$ (see Example 4 ) can be broken down into the following six subsequences:

$$
\begin{array}{rrrrr}
1, & 7, & -15, & 23, & \ldots \\
1, & -9, & 17, & -25, & \ldots \\
2, & -2, & 2, & -2, & \ldots \\
3, & -11, & 19, & 27, & \ldots \\
5, & -13, & 21, & -29, & \ldots \\
-2, & 2, & -2, & 2, & \ldots
\end{array}
$$

In absolute value, it appears that four of the six subsequences are growing linearly with difference 8, with the exception of the first two terms of the first subsequence, and the other two subsequences are constant in absolute value and hence bounded. We have

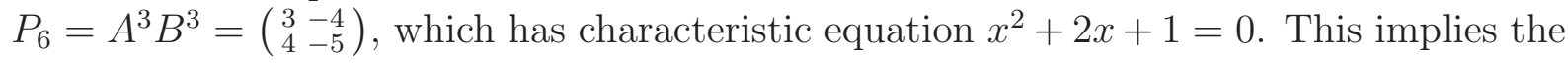


recurrence $t_{n}=-2 t_{n-1}-t_{n-2}$, which fits our data, even the exception. Solving, we get a double eigenvalue $\lambda_{1}=-1$, which by Proposition 8 implies linear growth, but we must also consider the third and sixth subsequences. Here we have $\lambda_{1}=-1$ and $a_{1}=-a_{2}$, which again by Proposition 8 implies bounded growth.

The periodic sequence for the cycle $\sigma_{3}=(++-)$ is broken down into three subsequences as follows:

$$
\begin{array}{rrrrrrr}
1, & 3, & 1, & 3, & 1, & 3, & \ldots \\
1, & -1, & 1, & -1, & 1, & -1, & \ldots \\
2, & 2, & 2, & 2, & 2, & 2, & \ldots
\end{array}
$$

Each of these sequences is clearly bounded. The product matrix in this case is $P_{3}=$ $A^{2} B=\left(\begin{array}{cc}1 & 0 \\ 2 & -1\end{array}\right)$, which has characteristic equation $x^{2}-1=0$. The corresponding recurrence is $t_{n}=t_{n-2}$, which is satisfied by all three subsequences. The eigenvalues of the characteristic equation are $\lambda_{1}=1, \lambda_{2}=-1$ and Theorem 10 tells us distinct roots of unity imply bounded growth.

We can extend this example by considering what happens when we double the coefficient cycle. Let $\sigma_{6}=(++-++-)$. We know it must behave the same way as $\sigma_{3}=(++-)$ because it generates the same periodic coefficient sequence. This time we obtain six subsequences, each of which is constant, and product matrix $P_{6}=P_{3}^{2}=I$, with characteristic equation $x^{2}-2 x+1=0$, recurrence $t_{n}=2 t_{n-1}-t_{n-2}$ and double eigenvalue $\lambda_{1}=1$. The double eigenvalue would suggest linear growth, but again this falls into the exceptional case because each of our subsequences has $a_{1}=a_{2}$, ensuring bounded growth.

Linear growth requires a double eigenvalue of \pm 1 , and so there are only two options for the recurrence, namely

$$
t_{n}= \pm 2 t_{n-1}-t_{n-2} .
$$

It is clear that if $a_{1}= \pm a_{2}$ (depending on the value of $\lambda_{1}$ ), the sequence will remain constant in absolute value.

The following known result characterizes sequences with bounded growth.

Proposition 14. A periodic coefficient sequence for $\sigma_{n}$ has bounded growth if and only if it is periodic with some finite period length $l=n k$.

Our proof in [16] uses the fact that any product matrix has one of three possible Jordan normal forms over $\mathbb{C}$, depending on its eigenvalues, to show that a periodic coefficient sequence has bounded growth if and only if the associated product matrix has finite order $k$. This is equivalent to repeating the coefficient cycle $k$ times to create a new coefficient cycle of length $n k$, which gives us a list of constant subsequences. McGuire [13] makes use of the pigeonhole principle to prove this result.

We can now define the growth of a periodic coefficient sequence, and further, determine the exact growth rate in the case of exponential growth. 
Definition 15. We say that a periodic coefficient sequence $\left\{t_{i}\right\}$ for $\sigma_{n}$ grows exponentially if all $n$ subsequences grow exponentially, we say it grows linearly if at least one subsequence grows linearly and it is bounded if all $n$ subsequences are bounded.

Proposition 16. The growth rate of an exponentially growing periodic coefficient sequence $\left\{t_{i}\right\}$ for $\sigma_{n}$ is $\left|\lambda_{1}\right|^{\frac{1}{n}}$, where $\lambda_{1}$ is the dominant eigenvalue of the corresponding product matrix $P_{n}$.

Proof. Theorem 10 and Corollary 12 together tell us that the dominant eigenvalue $\lambda_{1}$ exists and gives us the growth rate of any of the $n$ subsequences which are formed by considering every $n^{\text {th }}$ term in the periodic coefficient sequence. Taking the $n^{\text {th }}$ root of this growth rate allows us to determine the growth rate of the periodic coefficient sequence term by term.

\section{Classification of Periodic Coefficient Sequences}

The following results relate the parity of the term $u$, given in Equation (10), with the divisibility of $n$.

Theorem 17. For a product matrix $P_{n}$ with trace $u$,

$$
\begin{aligned}
\text { u even } & \Longleftrightarrow 3 \mid n, \\
\text { u odd } & \Longleftrightarrow 3 \nmid n .
\end{aligned}
$$

Proof. Applying the modulo 2 homomorphism

$$
\bmod _{2}: \mathrm{SL}(2, \mathbb{Z}) \rightarrow \mathrm{SL}(2, \mathbb{Z} / 2 \mathbb{Z}),
$$

we see that our matrices $A$ and $B$ have equivalent images. The images of powers of $A$ behave in a cyclical fashion, rotating through $A,\left(\begin{array}{ll}1 & 1 \\ 1 & 0\end{array}\right)$ and $I$, for $n \equiv 1,2,0(\bmod 3)$ respectively. The trace value $u$ therefore behaves as required.

Theorem 18. For a product matrix $P_{n}$ with even trace $u$ we have the further distinction

$$
\begin{aligned}
& u \equiv 0 \quad(\bmod 4) \Longleftrightarrow n \equiv 3 \quad(\bmod 6), \\
& u \equiv 2 \quad(\bmod 4) \Longleftrightarrow n \equiv 0 \quad(\bmod 6) .
\end{aligned}
$$

The proof is similar to that of Theorem 17, taking matrix entries modulo 4 instead. The following theorem, which is one of our main results, gives the connection between trace value and type of growth. We use the fact that the trace of a matrix is equal to the sum of its eigenvalues, i.e., $u=\lambda_{1}+\lambda_{2}$.

Theorem 19. Given a product matrix $P_{n}$ with $n$ odd and $P_{n} \neq \pm I$, the growth of the corresponding periodic coefficient sequence is

$$
\begin{aligned}
\text { exponential } & \Longleftrightarrow u \neq 0, \\
\text { bounded } & \Longleftrightarrow u=0,
\end{aligned}
$$


and if $n$ is even the sequence growth is

$$
\begin{aligned}
\text { exponential } & \Longleftrightarrow|u|>2, \\
\text { linear } & \Longleftrightarrow|u|=2, \\
\text { bounded } & \Longleftrightarrow|u|=1 .
\end{aligned}
$$

If $P_{n}= \pm I$, growth is bounded.

Proof. We have seen in Theorem 10 that for $P_{n}= \pm I$, growth is not linear but bounded, despite the fact that $u= \pm 2$, and from here on we will exclude this case. Recall that the characteristic equation of a product matrix has form $x^{2}-u x \pm 1$. If we first consider the general $n$ odd case, our characteristic equation has the form $x^{2}-u x-1$, giving eigenvalues

$$
\lambda_{1}, \lambda_{2}=\frac{u \pm \sqrt{u^{2}+4}}{2} .
$$

We know from Theorem 10 and Definition 15 that the growth of a periodic coefficient sequence is bounded if and only if the eigenvalues are distinct roots of unity. Using the fact that the discriminant is always positive (and hence the eigenvalues cannot be complex), we have boundedness if and only if $\lambda_{1}=1, \lambda_{2}=-1$. In turn, this occurs if and only if $u=\lambda_{1}+\lambda_{2}=0$. Since the radical is always positive, we can never have a double root, i.e., growth is never linear, by Theorem 10. Therefore, since it is the only other option, we must have exponential growth if and only if $u \neq 0$.

If we now consider the $n$ even case, our characteristic equation has form $x^{2}-u x+1$, giving eigenvalues

$$
\lambda_{1}, \lambda_{2}=\frac{u \pm \sqrt{u^{2}-4}}{2} .
$$

We know from Theorem 10 that the growth of our sequence is linear if and only if the eigenvalues are both equal to 1 or -1 . In turn, this occurs if and only if $u=\lambda_{1}+\lambda_{2}= \pm 2$. For $n$ even we have $\operatorname{det}\left(P_{n}\right)=1$, and therefore $\lambda_{1} \lambda_{2}=1$. If $\lambda_{1}, \lambda_{2}$ are distinct roots of unity, they must be complex, in which case we need $u^{2}-4<0$. Therefore the only possibilities for bounded growth are for $u=0, \pm 1$. But we have seen in Theorem 18 that $u$ can take on the value 0 only when $n \equiv 3(\bmod 6)$, in which case $n$ is odd. Therefore bounded growth must occur only when $u= \pm 1$. Conversely, when $u=1,-1$ we get eigenvalues $\frac{1 \pm \sqrt{-3}}{2}$ and $\frac{-1 \pm \sqrt{-3}}{2}$ respectively, which are pairs of distinct roots of unity and hence growth is bounded by Theorem 10. The remaining $u$ values, i.e., $|u|>2$ therefore correspond to sequences with remaining growth type, exponential growth.

Combining previous results we have the following corollary.

Corollary 20. Given a product matrix $P_{n} \neq \pm I$ we have the following results about the growth of the corresponding periodic coefficient sequence:

$$
\begin{aligned}
\text { linear } & \Rightarrow 6 \mid n, \\
n \text { or } u \text { odd } & \Rightarrow \text { bounded or exponential. }
\end{aligned}
$$


If $P_{n}= \pm I$, we have $6 \mid n$. Further, if $n$ is odd we have

$$
\begin{aligned}
\text { bounded } & \Rightarrow 3 \mid n, \\
3 \nmid n & \Rightarrow \text { exponential. }
\end{aligned}
$$

Proof. By Theorem 19 linear growth occurs only when $n$ is even and $|u|=2$. Theorem 17 tells us that $u$ is even if and only if $3 \mid n$, hence $6 \mid n$. Therefore, if $6 \nmid n$ then growth is not linear. In other words if $n$ or $u$ is odd (i.e., $2 \nmid n$ or $3 \nmid n$ ) then growth is bounded or exponential. In the $P_{n}= \pm I$ case, the proof follows as above because $\operatorname{det}( \pm I)=1$, implying $n$ is even.

For $n$ odd, Theorem 19 tells us that bounded growth implies $u=0$. Theorem 17 then tells us that for $u$ even, $3 \mid n$. The second statement is simply the contrapositive of the first, where we have used the fact from Theorem 19 that when $n$ is odd, if growth is not bounded, it must be exponential.

We have looked at the connection between the trace of a product matrix and growth type of the corresponding periodic coefficient sequence and now we will consider the connection between the order of a product matrix and growth type. The following definition can be found in Weinstein [18, p. 84].

Definition 21. The projective special linear group, $\operatorname{PSL}(2, \mathrm{~F})$, for a field $F$ is defined as the quotient group

$$
\operatorname{PSL}(2, F):=\mathrm{SL}(2, F) / \mathrm{Z}(\mathrm{SL}(2, \mathrm{~F})),
$$

where $\mathrm{SL}(2, F)$ is the special linear group and $\mathrm{Z}(\mathrm{SL}(2, F))$ is its center.

We can similarly define this group over a ring $R$.

The following result can be found in $[18$, p. 83]. We are interested in the group $G \leqslant \mathrm{~S}^{*} \mathrm{~L}(2, \mathbb{Z})$ of product matrices, where $\mathrm{S}^{*} \mathrm{~L}(2, \mathbb{Z})$ has corresponding projective group $\mathrm{PS}^{*} \mathrm{~L}(2, \mathbb{Z})=\mathrm{S}^{*} \mathrm{~L}(2, \mathbb{Z}) / \mathrm{Z}\left(\mathrm{S}^{*} \mathrm{~L}(2, \mathbb{Z})\right)$.

Proposition 22. Let $R$ be a commutative ring with identity. Then $\mathrm{Z}(\mathrm{SL}(2, R))=\{ \pm I\}$.

Therefore $\mathrm{Z}(\mathrm{SL}(2, \mathbb{Z}))=\{ \pm I\}$, and similarly, $\mathrm{Z}\left(\mathrm{S}^{*} \mathrm{~L}(2, \mathbb{Z})\right)=\{ \pm I\}$. Since $G$ is a subgroup of $\mathrm{S}^{*} \mathrm{~L}(2, \mathbb{Z})$, we can define the group $P G$ which is a subgroup of $\operatorname{PS}{ }^{*} \mathrm{~L}(2, \mathbb{Z})$ and has elements $[M]=\{ \pm M\}$, where $M \in G$. The identity of all of these projective groups is $[I]=\{ \pm I\}=1$.

The following results are taken from [18, p. 89].

Theorem 23. Let $[M] \in \operatorname{PS}^{*} \mathrm{~L}(2, \mathbb{Z})$. Then $\operatorname{ord}([M])=2$ if and only if $\operatorname{tr}(M)=0$.

This result is proved in $[18$, p. 89] for matrices $[M] \in \operatorname{PSL}(2, F)$ with $\operatorname{char}(F) \neq 2$. Since it is therefore true for matrices with entries in $\mathbb{R}$, it must also be true for matrices with strictly integer entries, and the result can be similarly proved for matrices with determinant -1 . 
Corollary 24. Let $M \in \mathrm{S}^{*} \mathrm{~L}(2, \mathbb{R})$ with $\operatorname{tr}(M)=0$. Then

$$
\begin{aligned}
\operatorname{det}(M)=1 & \Longleftrightarrow M^{2}=-I \Longleftrightarrow \operatorname{ord}(M)=4, \\
\operatorname{det}(M)=-1 & \Longleftrightarrow M^{2}=I \Longleftrightarrow \operatorname{ord}(M)=2 .
\end{aligned}
$$

The majority of the proof follows from the proof of Theorem 23. We must also look at the general form of the matrix $M^{4}$, where $\operatorname{tr}(M)=0$.

The following theorem also comes from a result of Weinstein [18, p. 90], who proved it for $\operatorname{PSL}(2, F)$ for a field $F$ with $\operatorname{char}(F) \neq 3$.

Theorem 25. Let $[M] \in \operatorname{PSL}(2, \mathbb{Z})$. Then $\operatorname{ord}([M])=3$ if and only if $\operatorname{tr}(M)= \pm 1$.

This theorem is not true in general for $\operatorname{PS}^{*} \mathrm{~L}(2, \mathbb{Z})$. Again we can distinguish between the two possible orders, using the proof of Theorem 25 .

Corollary 26. For a matrix $M \in \mathrm{SL}(2, F)$ we have

$$
\begin{aligned}
\operatorname{tr}(M)=1 & \Longleftrightarrow M^{3}=-I \Rightarrow \operatorname{ord}(M)=6, \\
\operatorname{tr}(M)=-1 & \Longleftrightarrow M^{3}=I \Longleftrightarrow \operatorname{ord}(M)=3 .
\end{aligned}
$$

If we consider our specific group $G=\langle A, B\rangle$ of product matrices $P_{n}$, we obtain the following results.

Proposition 27. For a product matrix $P_{n}$, we have that $\operatorname{tr}\left(P_{n}\right)=0$ if and only if $\operatorname{ord}\left(P_{n}\right)=2$.

Proof. If $\operatorname{ord}\left(P_{n}\right)=2$ we have by Theorem 23 that $\operatorname{tr}\left(P_{n}\right)=0$. Conversely, suppose that $\operatorname{tr}\left(P_{n}\right)=0$. We want to discount the possibility in Theorem 23 of $\operatorname{ord}\left(P_{n}\right)=4$. By Theorem 18, we have that if $\operatorname{tr}\left(P_{n}\right)=0$ then $n \equiv 3(\bmod 6)$. But by Corollary 24 , since $n$ is odd (i.e., $\operatorname{det}\left(P_{n}\right)=-1$ ), we must have $\operatorname{ord}\left(P_{n}\right)=2$.

Corollary 28. For a product matrix $P_{n} \neq \pm I$ with $\operatorname{det}\left(P_{n}\right)=1$ :

$$
\operatorname{ord}\left(\left[P_{n}\right]\right)=3 \Longleftrightarrow \text { bounded, }
$$

For a product matrix $P_{n} \neq \pm I$ with $\operatorname{det}\left(P_{n}\right)=-1$ :

$$
\operatorname{ord}\left(P_{n}\right)=2 \Longleftrightarrow \text { bounded. }
$$

Proof. Suppose $\left[P_{n}\right]$ has order 2 or 3 . The note after Proposition 14 tells us that a product matrix with finite order corresponds to bounded growth. Now suppose growth is bounded. Theorem 19, for the $n$ even case (i.e., $\operatorname{det}\left(P_{n}\right)=1$ ), tells us that $|u|=1$. By Theorem 25 , the order of $\left[P_{n}\right]$ must be 3 . If we have $\operatorname{det}\left(P_{n}\right)=-1$, Theorem 19 tells us that growth is bounded if and only if $\operatorname{tr}(M)=0$, but by Proposition $27, \operatorname{tr}\left(P_{n}\right)=0$ if and only if $\operatorname{ord}\left(P_{n}\right)=2$.

We can now completely characterize bounded sequences for any value of $n$. 
Corollary 29. For any product matrix $P_{n} \neq \pm I$,

$$
\text { bounded } \Longleftrightarrow \operatorname{ord}\left(P_{n}\right)=2,3 \text { or } 6 \text {. }
$$

Proof. This follows directly from Corollary 28 , using the fact that ord $\left(\left[P_{n}\right]\right)=3$ implies $\operatorname{ord}\left(P_{n}\right)=3$ or 6 .

Note that it is known that any element $M \in \mathrm{GL}(2, \mathbb{Z})$ of finite order (i.e., having bounded growth for $P_{n}$ ) has order 1, 2, 3, 4 or 6 . The proof can be found in [12], for example.

McGuire [13] gives a proof of Corollary 29 using eigenvalues in polar coordinates. In his proof he uses the facts that if $\lambda_{1}, \lambda_{2}= \pm 1$ then $\operatorname{ord}\left(P_{n}\right)=2$ (in which case we have $\operatorname{det}\left(P_{n}\right)=-1$ and $\left.\operatorname{tr}\left(P_{n}\right)=0\right)$, and if $\operatorname{tr}\left(P_{n}\right)= \pm 1$ for $P_{n}$ with finite order, then $\operatorname{ord}\left(P_{n}\right)=6,3$ respectively. He also gives the following necessary condition for sequences to be periodic (i.e., bounded, by Proposition 14). In our terminology, given a coefficient cycle of length $n$ determining a bounded periodic coefficient sequence, we must have

$$
\operatorname{ord}\left(P_{n}\right) \cdot n \equiv 0 \quad(\bmod 3) .
$$

The converse of this statement is not true, however. McGuire's result is implicit in our previous results on order and trace. Interestingly, in [14] he generalizes this result to random $m$-nacci $\left(m^{\text {th }}\right.$ order) sequences. Instead of forming periodic coefficient sequences using coefficient cycles of \pm signs, he uses size- $n$ sets of $(m-1)$-tuples of \pm signs. Using products of $m \times m$ matrices he shows that for a bounded periodic coefficient sequence, we must have

$$
\operatorname{ord}\left(P_{n}\right) \cdot n \equiv 0 \quad(\bmod m+1)
$$

\section{Approximating Viswanath's Constant}

One way to approximate Viswanath's constant is to generate a long random Fibonacci sequence and calculate its exponential growth using Definition 7. This random sampling was used by Viswanath [19], where a sequence of length one million was shown to have growth rate 1.132 and reached values of over $10^{50,000}$. This still only gives two decimal places of accuracy! We can increase the accuracy in this random sampling by taking averages of growth rates. Following, are the averages of the growth rates of 20 random Fibonacci sequences of length 40002, using the definition of growth given in Proposition 16 with $n=40,000$. These values actually appear to be slightly more accurate than the

\begin{tabular}{|c|c|c|}
\hline 1.131885031 & 1.131973850 & 1.131740524 \\
\hline 1.132081608 & 1.132389153 & 1.1323296404 \\
\hline
\end{tabular}

previous approximation, despite the smaller sequence length. It is thus quite remarkable that Viswanath and Bai were able to find 8 and 13 correct decimals respectively.

Also, instead of generating sequences randomly, it is possible to form periodic coefficient sequences for all possible length- $n$ coefficient cycles, and take the arithmetic mean 
of their growth rates. This generates a set of periodic coefficient sequences (of infinite length), some of which will approximate random Fibonacci sequences as $n$ gets large. By letting $n \rightarrow \infty$, the average growth rate should effectively estimate Viswanath's constant. For $n=20$ there are over half a million branches and the average growth rate calculated is 1.18 (see Hayes [9]). Figure 1 shows the average values of the growth rates of all possible random Fibonacci sequences for $\sigma_{n}$ with $n \leqslant 19$. For large $n$ this gives us the following approximation:

$$
e^{\gamma_{f}} \approx \frac{1}{2^{n}} \sum_{\text {all } P_{n}}\left(\frac{|| \operatorname{tr}\left(P_{n}\right)\left|+\sqrt{\operatorname{tr}\left(P_{n}\right)^{2} \pm 4}\right|}{2}\right)^{\frac{1}{n}}
$$

where the bracketed term is the absolute value of the largest eigenvalue, as given in Equations (11) and (12).

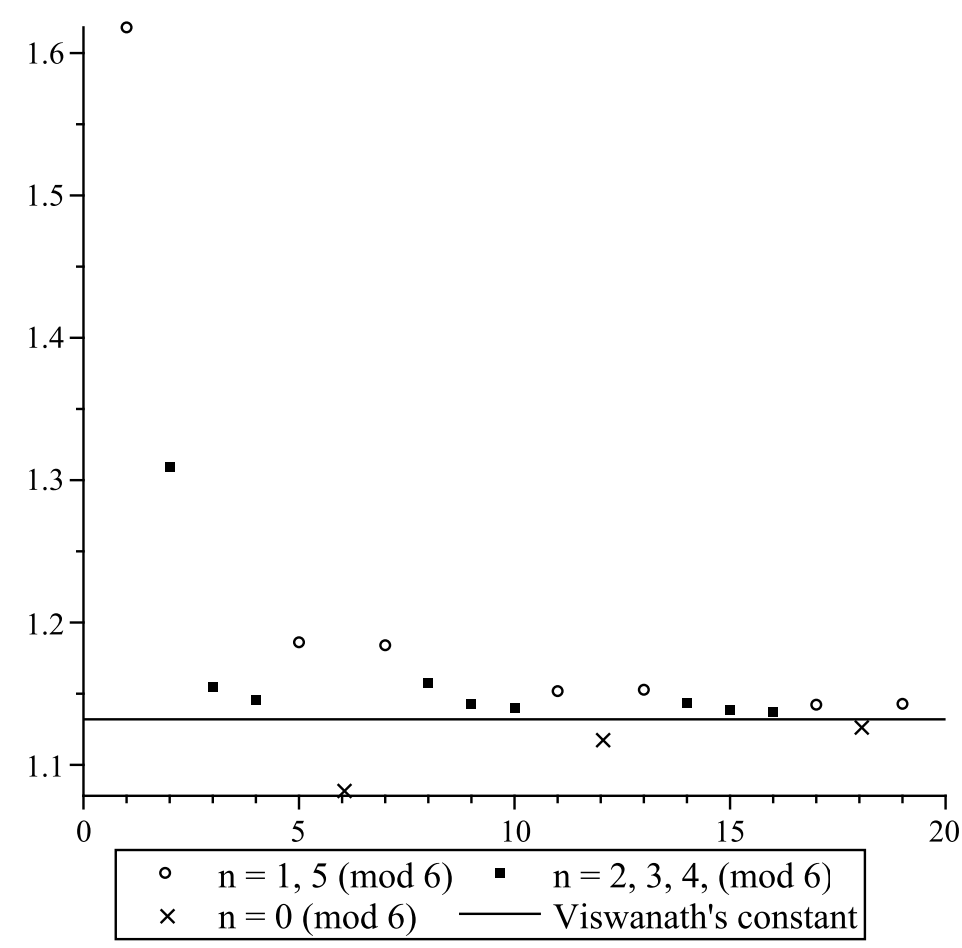

Figure 1: Average values of growth rates.

It is easily recognizable from Figure 1 that the growth rate follows a pattern for values of $n$ (mod 6$)$. We can partially explain this pattern by considering some of the previously gathered results on growth type. We have seen in Corollary 20 that linear growth occurs only when $6 \mid n$. This growth rate here is 1 and so we expect that the growth rate for $n \equiv 0$ (mod 6) will be lower than the average growth rate for other values of $n$, as evidenced by the x's in Figure 1. Similarly, for $n$ odd, Corollary 20 tells us that if $3 \nmid n$, growth is 
exponential. Therefore, for $n \equiv 1,5(\bmod 6)$, the growth rates must be strictly greater than 1 and we might expect the average of growth rates for such $n$ to be raised, as evidenced by the circles in Figure 1.

We can simplify our average growth rate calculation by noticing that, for large values of $u$, we have the approximation $\left|\operatorname{tr}\left(P_{n}\right)\right|=|u| \approx\left|\lambda_{1}\right|$, and so Equation (13) becomes

$$
e^{\gamma_{f}} \approx \frac{1}{2^{n}} \sum_{\text {all } P_{n}}|u|^{\frac{1}{n}} .
$$

We can now reduce the size of this calculation (or that in (13)) by dividing up the number of cases we need to consider into equivalence classes. Proposition 11 told us that rotating terms in a product matrix leaves trace and determinant unchanged. Similarly, we have the following two propositions, which can be easily proven using properties of matrices, or continuant polynomials (for a good introduction to continuants, see [8]).

Proposition 30. Given a coefficient cycle $\sigma_{n}=\left(s_{1}, \ldots, s_{n}\right)$ with $n \geqslant 1$ and corresponding product matrix $P_{n}$, reversing the terms in the cycle gives $\sigma_{n}^{\prime}=\left(s_{n}, \ldots, s_{1}\right)$ with corresponding product matrix $P_{n}^{\prime}$, where $P_{n}^{\prime}$ is the transpose of $P_{n}$ and hence has the same characteristic equation and growth rate as $P_{n}$.

Proposition 31. Given a coefficient cycle $\sigma_{n}=\left(s_{1}, \ldots, s_{n}\right)$, with $n \geqslant 1$, switching each term $s_{n}$ from + to - or vice versa gives us a new cycle $\sigma_{n}^{\prime}$ with corresponding product matrix having the same growth rate as the original product matrix.

Further, if we reduce or extend the period by a multiple of the primitive cycle $\sigma_{p}$, i.e., the smallest repeating cycle comprising the coefficient cycle $\sigma_{n}$, the growth rate is unchanged. We can now define an equivalence class on our coefficient cycles as follows.

Definition 32. We write $\sigma_{n} \sim \tau_{d}$ if the cycle $\sigma_{n}=\left(s_{1}, \ldots, s_{n}\right)$, where $n \geqslant 1$, can be transformed into the cycle $\tau_{d}=\left(t_{1}, \ldots, t_{d}\right)$ by applying any finite combination of the following operations:

1. reversal: $\left(s_{1}, \ldots, s_{n}\right) \rightarrow\left(s_{n}, \ldots, s_{1}\right)$;

2. rotation: $\left(s_{1}, \ldots, s_{n}\right) \rightarrow\left(s_{n-k+1}, \ldots, s_{n}, s_{1}, \ldots, s_{n-k}\right)$;

3. negation: $\left(s_{1}, \ldots, s_{n}\right) \rightarrow\left(-s_{1}, \ldots,-s_{n}\right)$;

4. period reduction or extension: $\left(s_{1}, \ldots, s_{n}\right) \rightarrow\left(s_{1}, \ldots, s_{d}\right)$, where $p \mid d$, i.e., $\sigma_{n}$ is reduced or extended by a multiple of its primitive cycle $\sigma_{p}$.

Instead of using a repeated coefficient cycle to generate a periodic coefficient sequence, we can picture it as a loop which is continually traversed. This necklace is a string of $n$ characters of $q$ different types (commonly thought of as $n$ beads of $q$ colors) which is unchanged by rotation (see, for e.g., [8]). Similarly, a bracelet is unchanged by rotation and reversal. The number of equivalence classes, i.e., primitive (non-repeating) two-colored 
bracelets with color swapping (negation), of coefficient cycles $\sigma_{n}$ is given by the sequence (see [20, A000046])

$$
1,1,1,1,2,3,5,8,14,21,39,62,112, \ldots
$$

for $n \geqslant 0$. There are combinatorial formulas for the number of equivalence classes [20, A000046], for bracelets with color swapping [20, A000011], for necklaces with color swapping [20, A000013], and for necklaces [20, A000031].

\section{Conclusion}

In summary, the main results of this paper are the classification of the growth of periodic coefficient sequences according to eigenvalue (Theorem 10) and trace (Theorem 19), the explicit computation of the exponential growth rate of a periodic coefficient sequence (Proposition 16) and the use of equivalence classes of sequences having the same growth rate (Definition 32 ).

Our original intent was to try to shed some light on Viswanath's constant by determining the number of equivalence classes, the size of each equivalence class, and the growth type/rate of each equivalence class. It may then have been possible to combine this information into a formula for the average growth rate of all periodic coefficient sequences for $\sigma_{n}$. By letting $n \rightarrow \infty$, we would in theory be computing Viswanath's constant. This approach turned out to be quite difficult. We have found the number of equivalence classes for period length $n$, and a way to determine the growth rate of each, but finding the size of each class and constructing a formula are complex problems.

We have evidence of a second characterization of growth type, similar to that in Theorem 19, based on continued fractions. For a coefficient cycle $\sigma_{n}=\left(s_{1}, s_{2}, \ldots, s_{n}\right)$, with $s_{i} \in\{+,-\}$, we consider the corresponding non-simple periodic continued fraction $\gamma_{n}=\left[\hat{s}_{1}, \hat{s}_{2}, \ldots, \hat{s}_{n}\right]$, where $\hat{s}_{j} \in\{1,-1\}$.

The study of random Fibonacci sequences is relatively new, and filled with deep and

interesting results. In [15], Trefethen is quoted as saying: "Looking for patterns and trends among such sequences of numbers can be a fascinating pastime."

\section{Acknowledgements}

The author would like to thank Dr. Karl Dilcher for his support during the writing of this paper, and Dr. Keith Johnson for providing Proposition 6.

\section{References}

[1] Zai-Qiao Bai. On the cycle expansion for the Lyapunov exponent of a product of random matrices. J. Phys. A, 40(29):8315-8328, 2007.

[2] Zai-Qiao Bai. An infinite transfer matrix approach to the product of random $2 \times 2$ positive matrices. J. Phys. A, 42(1):015003, 2009. 
[3] Prem N. Bajaj. Equality of limits in ratio and root tests. Mathematics Magazine, 71(4):299, 1998.

[4] E. R. Colman, G. J. Rodgers. The resistance of randomly grown trees. J. Phys. A, 44(50):505001, 2011.

[5] Keith Devlin. New mathematical constant discovered: Descendant of two thirteenth century rabbits. Devlin's Angle, http://www.maa.org/external_archive/devlin/devlin_3_99.html, 1999.

[6] Mark Embree, Lloyd N. Trefethen. The growth and decay of random Fibonacci sequences. Proc. R. Soc. Lond. Ser. A. Math. Phys. Eng. Sci., 455(1987):2471-2485, 1999.

[7] Harry Furstenberg. Noncommuting random products. Trans. Amer. Math. Soc., 108(3):377-428, 1963.

[8] Ronald Graham, Donald Knuth, and Oren Patashnik. Concrete mathematics: A foundation for computer science, 2nd ed. Addison-Wesley, Boston, MA, 1994.

[9] Brian Hayes. The vibonacci numbers. Amer. Scientist, 87(4):296-301, 1999.

[10] Élise Janvresse, Benoît Rittaud, and Thierry de la Rue. How do random Fibonacci sequences grow? Probab. Theory and Related Fields, 142(3-4):619-648, 2008.

[11] Élise Janvresse, Benoît Rittaud, and Thierry de la Rue. Almost-sure growth rate of generalized random Fibonacci sequences. Ann. Inst. Henri Poincaré Probab. Stat., 46(1):135-158, 2010.

[12] James Kuzmanovich, Andrey Pavlichenkov. Finite groups of matrices whose entries are integers. Amer. Math. Monthly, 109(2):173-186, 2002.

[13] Trevor McGuire. On the periodicity of certain recursive sequences. Fibonacci Quart., 46/47(4):350-355, 2008/2009.

[14] Trevor McGuire. Generalizations of the periodicity of certain recursive sequences. Fibonacci Quart., 48(2):175-181, 2010.

[15] Ivars Peterson. Fibonacci at random: Uncovering a new mathematical constant. Science News, 155(24):376, 1999.

[16] Karyn McLellan Periodic coefficients and random Fibonacci sequences. Dalhousie University, http://hdl . handle.net/10222/15381, 2012.

[17] S. Vajda. Fibonacci $\&$ Lucas numbers, and the golden section. Ellis Horwood Limited, Chichester, England, 1989.

[18] Michael Weinstein. Examples of gropus. Polygonal Publishing House, Passaic, N.J., 1977.

[19] Divakar Viswanath. Random Fibonacci sequences and the number 1.13198824... Math. Comp., 69(231):1131-1155, 1999.

[20] Neil J. A. Sloane. Online encyclopedia of integer sequences. http://oeis.org/, 2013. 